\title{
Transient Thermoelastic Analysis of a Cylinder Having a Varied Coefficient of Thermal Expansion
}

\author{
Olga Shypul1*, Vitalii Myntiuk ${ }^{1}$ \\ ${ }^{1}$ Technology of Aircraft Manufacturing Department, Faculty of Aircraft Building, National Aerospace University \\ "Kharkiv Aviation Institute", 61070 Kharkiv, 17 Chkalov str., Ukraine \\ * Corresponding author, e-mail: o.shipul@khai.edu
}

Received: 23 July 2019, Accepted: 12 August 2020, Published online: 21 September 2020

\begin{abstract}
This paper is concerned with the mathematical modeling of transient thermal elastic problem involving a layered cylinder with a varied coefficient of thermal expansion and powered by a heat flux from an external surfaces. All material's properties are the same for each cylinder's layers, besides the coefficient of linear thermal expansion which is varied and corresponds to hardened and unhardened layers. An obtained solution is a transient state of a heat transfer for the one-dimensional temperature change under the action of heat flux in continuous time. Cumbersome analytical solutions are converted into simple approximation. They are used to solve the inverse problems of the thermal stressed state-determining the time of action of the heat flux to achieve the specified maximum temperature or stress. Some numerical results for the stress distributions are shown in figures.
\end{abstract}

Keywords

layered cylinder, heat transfer, transient state, thermal elastic analysis

\section{Introduction}

Due to modern technical challenges either the implementation of new technologies or materials in modern hightech manufacturing requires the solution of important problems of assessing the strength of the materials being processed. This is especially important for finishing technology of shock-wave treatment, such as ultrasonic hardening [1] or finishing by detonating gas mixtures (thermal deburring) [2], using the energy of heat flows, including high intensity. And here it is very important not only to accurately determine the operating heat fluxes [3], but also the ability of materials to perceive such heat loads.

A special direction in this problem is the issue of thermal elastic analysis of materials with different properties in thickness, for example, treatment metals with a pre-hardened surface layer (after cementation, quenching, etc.) by heat flow or polishing surfaces after dynamic coating with inherent variability of coverage [4]. It is known, that the variability of the coefficient of thermal expansion is the most significant for materials with the nonhomogeneity of mechanical properties. [5, 6]. So, a surge of thermal stresses occurs in the outer layers of the material when an intense energy flow acts. Therefore, during determination the stress-stain transient state it is absolutely necessary to take into account the stresses resulting from the temperature gradient.

The nonhomogeneous material described above is closest to the Functionally Graded Materials (FGMs) now is popular in academic circles. As a rule, the properties of such materials are set as a continuous function from one phase to another, implying that the sizes of the zones are comparable [7-9]. Many studies have been carried out within the framework proposed by Biot [10], the classical conventional coupled theory of thermal elasticity. The theory is based on the classical Fourier law of heat conduction. This approach involves the instantaneous distribution of heat in a solid, which is not practically implemented. Examples of analytical solutions of transient thermal stress problems can be found in [11-14].

Therefore, the main purpose of this paper is to obtain an exact analytical solution and present it in a foreseeable form for an investment in analyzing the results.

The object of the study is a regular zone of a cylindrical body with a thin outer layer. Used nonhomogeneous material emphasizes the difference in thermal expansion 
coefficients. Note that in FGMs materials are selected with similar values (for example, titanium alloy (Ti-6Al-4V), $\alpha=8.91 / \mathrm{MK}$ and zirconium oxide $\left.\left(\mathrm{ZrO}_{2}\right), \alpha=8.71 / \mathrm{MK}\right)$.

\section{Heat conduction problem}

\subsection{Heat distribution in an infinite cylinder}

Consider a two-layer cylinder with a varied coefficient of thermal expansion and powered by a heat flux from an external surface (see Fig. 1). Outer layer is thin and its thickness is represented by and respectively for dimensional and dimensionless description. The coefficients of linear thermal expansion of each layer are different and their values are constant. It is assumed that other mechanical properties in the both layers are the same. This corresponds to the formulation of the problem of the presence of a strengthened layer in a solid body.

The layered cylinder inner and outer radii are designated $\bar{R}-\bar{\delta}$ and $\bar{R}$ respectively for dimensional description as well as $R_{1}$ and 1 for dimensionless description. Moreover, three important points are highlighted in the depth of the cylinder: on the surface $r=1$, in the middle of the radius $r=0.5$ and in the center of the cylinder $r=0$.

The well-known solution of the problem of heat propagation in an infinite cylinder when exposed to a heat flux [15] in dimensionless quantities has the form:

$$
T=r^{2} / 2+2 t-1 / 4-2 \sum_{n=1}^{\infty} \frac{J_{0}\left(\mu_{n} r\right)}{\mu_{n}^{2} J_{0}\left(\mu_{n}\right) \exp \left(\mu_{n}^{2} t\right)},
$$

here:

- $T=\left(\bar{T}-\bar{T}_{0}\right) K / \varphi \bar{R}$ - temperature change;

- $r=\bar{r} / \bar{R}$ - radial coordinate (Fig. 1);

- $t=a \bar{t} / \bar{R}^{2}-$ time;

- $\mu_{n}$-roots of the equation $J_{1}\left(\mu_{n}\right)=0$;

- $J_{0}, J_{1}$ - Bessel functions of the first kind of zero and first orders;

- $\bar{T}, \bar{T}_{0}-$ dimensional current and initial temperatures, $K$;

- $K$ - coefficient of thermal conductivity, $W \cdot m \cdot K^{-1}$;

- $\bar{t}$-dimensional time, $s ; \varphi$ - heat flux, $W \cdot m^{-2}$;

- $\bar{R}$ - outer radius, $m$;

- $a$-thermal diffusivity $m \cdot \mathrm{s}^{-1}$.

Variation of the temperature change in the time by heat flux exposure is shown in Fig. 2.

The maximum temperature occurring on the surface $(r=1)$, is determined using Eq. (1) by Eq. (2):

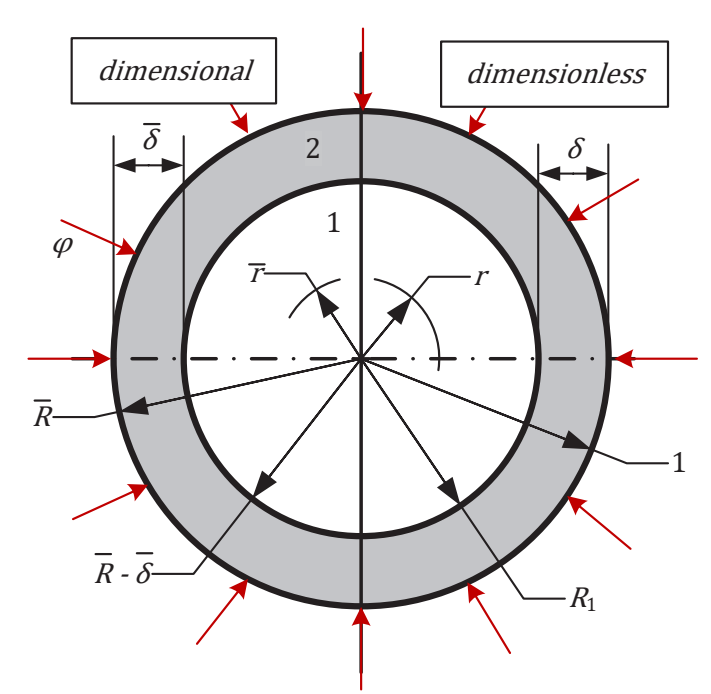

Fig. 1 The layered cylinder with a varied coefficient of thermal expansion

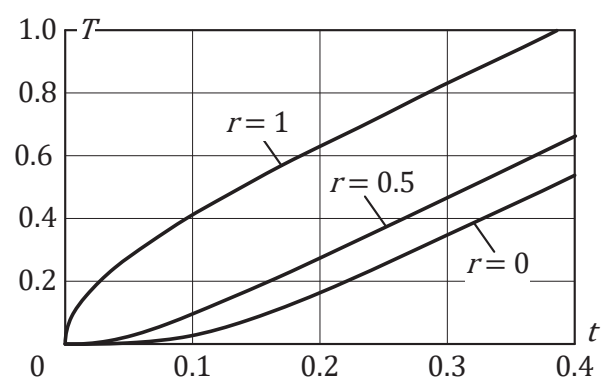

Fig. 2 Temperature variation of the time of heat flow exposure at three points of the cylinder

$T_{\max }=0.25+2 t-2 \sum_{n=1}^{\infty} \mu_{n}^{-2} \exp \left(-\mu_{n}^{2} t\right)$.

2.2 Approximation of the maximum temperature value From Fig. 2 and Eq. (2) it can be seen that the temperature dependence on time is nonlinear for small values of time $\bar{t}$. With increasing time, this dependence becomes linear.

Therefore, Eq. (2) can be approximated by Eq. (3):

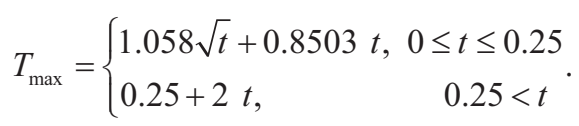

The error of the approximate value of the maximum temperature Eq. (3) relative to its exact value Eq. (2) does not exceed $5 \%$ in the entire range of time variation.

By approximation Eq. (3), it is easy to calculate the relative heating time of the cylinder to achieve the required temperature on its surface $T_{\max }$.

$t= \begin{cases}1.176 T_{\max }+0.7744 & \\ -\sqrt{0.5997+1.821 T_{\max }}, & T_{\max } \leq 0.75 . \\ 0.5 T_{\max }-0.125, & T_{\max }>0.75\end{cases}$ 


\section{Thermoelastic problem}

\subsection{Analytical solution}

Axisymmetric plane strain problem has a solution:

$\sigma_{r}=-r^{-2} \int r T d r+r^{-2} A+B$

$\sigma_{\theta}=-r^{-2} \int r T d r-T-r^{-2} A+B$,

$\sigma_{z}=\varepsilon_{z}-T+2 B v$

here:

- $\sigma=\bar{\sigma} K(1-v) /(E \alpha \varphi R)-$ dimensionless stresses;

- $\bar{\sigma}$-dimensional stresses, $\mathrm{Pa}$;

- $E$ - Young's modulus, Pa;

- $v$ - Poisson's ratio;

- $\alpha$-coefficient of linear thermal expansion;

- $\varepsilon_{z}=$ const - quantity of the axial strain.

The integration constants $A$ and $B$ are determined from boundary conditions.

Boundary conditions for a solid cylinder having an outer layer thickness $\bar{\delta}$ with a different coefficient of thermal expansion $k \alpha$ (Fig. 1) have a look:

$r=1 \rightarrow \sigma_{r 2}=0 ; r=0 \rightarrow \sigma_{r 1}<\infty$;

$r=R_{1} \rightarrow \sigma_{r 1}=\sigma_{r 2}$ and $\varepsilon_{\theta 1}=\varepsilon_{\theta 2}$.

The constant $\varepsilon_{z}$ characterize the longitudinal strain and is determined from the condition of self-balancing stresses:

$\int_{0}^{R_{1}} \sigma_{z 1} d r+\int_{R_{1}}^{1} \sigma_{z 2} d r=0$

In Eq. (6) and Eq. (7) the index "1" refers all values to the inside of a cylinder with a relative radius $R_{1}<1$, and the index "2" - to the outer layer with a relative thickness: $\delta=1-R_{1}$.

After determination the constants from Eq. (6) and Eq. (7) that are included in Eq. (5) and calculation the integral of temperature in Eq. (1), the final equation for calculating the stresses in both parts of the cylinder will take the following form (rather cumbersome):

$$
\begin{aligned}
& \sigma_{r 1}=\left(1-r^{2}+k_{1}\left(1-R_{1}^{2}\right)\left(R_{1}^{2}+8 t+4 T_{0}\right)\right) / 8 \\
& +2 \sum_{n=1}^{\infty} \frac{J_{1}\left(\mu_{n} r\right)+r k_{1} R_{1} J_{1}\left(\mu_{n} R\right)}{r \mu_{n}^{3} \exp \left(\mu_{n}^{2} t\right) J_{0}\left(\mu_{n}\right)}, \\
& \sigma_{\theta 1}=\left(1-3 r^{2}+k_{1}\left(1-R_{1}^{2}\right)\left(R_{1}^{2}+8 t+4 T_{0}\right)\right) / 8 \\
& +2 \sum_{n=1}^{\infty} \frac{\mu_{n} r J_{0}\left(\mu_{n} r\right)+J_{1}\left(\mu_{n} r\right)+r k_{1} R_{1} J_{1}\left(\mu_{n} R_{1}\right)}{r \mu_{n}^{3} \exp \left(\mu_{n}^{2} t\right) J_{0}\left(\mu_{n}\right)},
\end{aligned}
$$

$$
\begin{aligned}
& \sigma_{z 1}=k_{1} \delta\left(T_{0}+2 t+\frac{1}{4}-\delta(3-\delta)\right)+\frac{\left(1-3 r^{2}\right)}{6} \\
& +\sum_{n=1}^{\infty} \frac{C+2 J_{0}\left(\mu_{n} r\right)}{\mu_{n}^{2} \exp \left(\mu_{n}^{2} t\right) J_{0}\left(\mu_{n}\right)}, \\
& \sigma_{r 2}=\frac{\left(1-r^{2}\right)\left(k_{1} R_{1}^{2}\left(R_{1}^{2}+8 t-1+4 T_{0}\right)+k r^{2}\right)}{8 r^{2}} \\
& -2 \sum_{n=1}^{\infty} \frac{\left(1-r^{2}\right) k_{1} R_{1} J_{1}\left(\mu_{n} R_{1}\right)-k r J_{1}\left(\mu_{n} r\right)}{r^{2} \mu_{n}^{3} \exp \left(\mu_{n}^{2} t\right) J_{0}\left(\mu_{n}\right)}, \\
& \sigma_{\theta 2}=\frac{\left(k_{1}\left(r^{-2}+1\right) R_{1}^{2}\left(1-R_{1}^{2}-8 t-4 T_{0}\right)-k\left(3 r^{2}-1\right)\right)}{8} \\
& +2 \sum_{n=1}^{\infty} \frac{k_{1}\left(\left(1+r^{2}\right) R_{1} J_{1}\left(\mu_{n} R_{1}\right)-k r J_{1}\left(\mu_{n} r\right)-r \mu_{n} J_{0}\left(\mu_{n} r\right)\right)}{r^{2} \mu_{n}^{3} \exp \left(\mu_{n}^{2} t\right) J_{0}\left(\mu_{n}\right)}, \\
& \sigma_{z 2}=k_{1} R_{1}\left(\frac{\left(1-R_{1}^{2}\right)}{6}-T_{0}-2 t+\frac{1}{12}\right)+\frac{k\left(1-3 r^{2}\right)}{6} \\
& +\sum_{n=1}^{\infty} \frac{C+2 k J_{0}\left(\mu_{n} r\right)}{\mu_{n}^{2} \exp \left(\mu_{n}^{2} t\right) J_{0}\left(\mu_{n}\right)},
\end{aligned}
$$

To shorten the notation in Eqs. (8) to (13), it is indicated $k_{1}=k-1$, and:

$$
\begin{aligned}
& C=k J_{0}\left(\mu_{n}\right)\left(\pi \mathrm{H}_{1}\left(\mu_{n}\right)-2\right) \\
& +k_{1} r_{1}\left(\pi J_{1}\left(\mu_{n} r_{1}\right) \mathrm{H}_{0}\left(\mu_{n} r_{1}\right)-J_{0}\left(\mu_{n} r_{1}\right)\left(\pi \mathrm{H}_{1}\left(\mu_{n} r_{1}\right)-2\right)\right),
\end{aligned}
$$

where $H_{0}, H_{1}$ are struve functions zero and first orders.

To assess the influence of the thermal expansion coefficients for the two-layered model, the numerical results were obtained for the following data: $t=0.1, T_{0}=0$, outer layer thickness $\delta=0.2\left(R_{1}=0.8\right)$, the layer has one and half times greater coefficient of thermal expansion $k=1.5\left(k_{1}=0.5\right)$. This ratio of layer thicknesses is typical for metals after strengthening technologies.

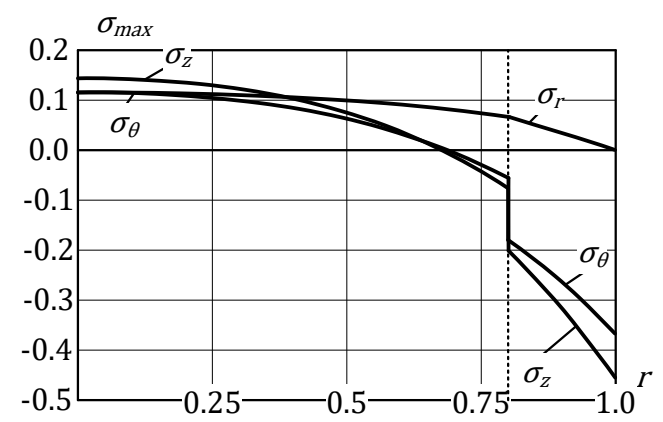

Fig. 3 Variation of thermal stresses along the radial direction 
Fig. 3 shows the variations of thermal stresses $\sigma_{z}, \sigma_{\theta}, \sigma_{r}$ in the transient state along the radial direction. The stress jump between the layers is due to the corresponding setting of the thermal expansion coefficient.

Different coefficients of thermal expansion of each layer cause jump in the tangential $\sigma_{\theta}$ and axial stresses $\sigma_{z}$. The radial stresses $\sigma_{r}$ are kinked. The value of maximum radial stresses (along the cylinder axis) is considerably lower than the maximum tangential and axial stresses (for the outer layer). The maximum tangential and axial stresses in the outer layer have the same level.

\subsection{Determination of maximum stresses}

The maximum stresses occur in the outer layer $r=1$, in addition, it can be noted that the outer layer is very thin. It is about relative thicknesses $\delta=0.01 \ldots 0.001$, so, it can be taken $\delta=0$.

With this assumption for $k<4$ and $t<1$, the error of determination of the maximum stresses do not exceed $2 \%$.

In view of this, Eq. (12) and Eq. (13) take the form:

$$
\begin{aligned}
& \sigma_{\theta \max }=2 k \sum_{n=1}^{\infty} \mu_{n}^{-2} \exp \left(\mu_{n}^{-2} t\right)-k_{1}\left(2 t+T_{0}\right)-\frac{k}{4}, \\
& \sigma_{z \max }=\sum_{n=1}^{\infty} \frac{\pi \mathrm{H}_{1}\left(\mu_{n}\right)+2 k_{1}}{\mu_{n}^{2} \exp \left(\mu_{n}^{2} t\right)}+k_{1}\left(1 / 12-T_{0}-2 t\right)-\frac{k}{3} .
\end{aligned}
$$

Variation of the maximum stresses over the time for different values $k$ is shown on Fig. 4. The figure clearly shows that for a solid cylinder $(k=1)$ the stresses reach a certain level and do not change with the heating time, but if $k>1$ the stresses grow indefinitely.

\subsection{Approximation of maximum stresses}

Maximum stresses (Eqs. (14) and (15)) (in general, like all others (Eqs. (8) to (13)) linearly depend on the relative coefficient of thermal expansion $k$. The type of stresses change over time is similar to the type of temperature change (see Fig. 4). In Fig. 4 it can be seen that temporal development of maximum stresses are nonlinear up to the time value $t<0.25$, and it becomes linear with increased time value.

Analytical solutions (Eqs. (14) and (15)) of studied task can be represented as following approximations (Eqs. (16) to (17)):

$$
\tilde{\sigma}_{\theta \max }=\left\{\begin{array}{ll}
2 t-(0.819 t+1.071 \sqrt{t}) k-(k-1) T_{0}, & t<0.25 \\
-\left(T_{0}+2 t\right)(k-1)-k / 4, & t \geq 0.25
\end{array},\right.
$$

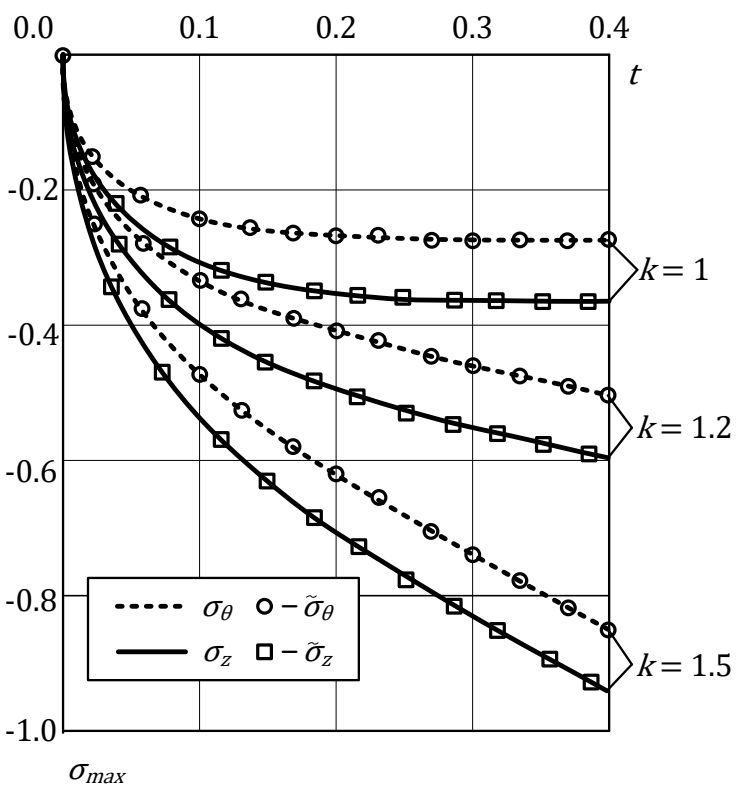

Fig. 4 The temporal development of maximum stresses: $\sigma_{\theta}, \sigma_{z}$ - analytical solutions, $\tilde{\sigma}_{\theta}, \tilde{\sigma}_{z}$ - approximated solutions

$$
\tilde{\sigma}_{z \max }=\left\{\begin{array}{ll}
(1.977-0.819 k) t & t<0.25 \\
-(1.071 k+0.167) \sqrt{t}(k-1) T_{0}, & \\
-(k-1)\left(T_{0}+2 t-1 / 12\right)-k / 3, & t \geq 0.25
\end{array}\right. \text {. }
$$

The error of these approximations does not exceed $5 \%$ over the whole range of time change $t$ and coefficient of thermal expansion $k$. The numerical results for approximations (Eqs. (16) and (17)) are shown in Fig. 4.

\subsection{Determination of heating time by maximum stresses}

Take into account the problem statement $(k>1)$, using the theory of maximum shear stresses $\left(\sigma_{f}=\sigma_{1}-\sigma_{3}\right)$ and having the temporal development of maximum stresses (see Fig. 4) it was defined the principal stresses $\sigma_{1}=0, \sigma_{2}=\sigma_{\theta}, \sigma_{3}=\sigma_{z}$ and finally the equivalent stresses $\sigma_{f}=-\sigma_{z}$.

For achievement a certain level of equivalent stresses $\sigma_{f}$ on outer side of the cylinder the required heating time can be determined from (Eq. (17)). To reduce the recording determined equation, the assumption of the absence of stresses in the body at the initial moment of time was taken. So, Eq. (18) for calculating the required heating become follow: 


$$
t= \begin{cases}\frac{\left(1.071 k+0.167-\sqrt{(3.276 k-7.908) \sigma_{f}+(1.071 k+0.167)^{2}}\right)^{2}}{(3.954-1.638 k)^{2}}, & \sigma_{f} \leq \frac{3 k}{4}-\frac{5}{12} \\ \frac{\left(12 \sigma_{f}-3 k-1\right)}{24(k-1)}, & \sigma_{f}>\frac{3 k}{4}-\frac{5}{12}\end{cases}
$$

In addition, Eq. (18) must be supplemented by two conditions that which eliminate singularity:

- if $k=1$ then $\sigma_{f} \leq 1 / 3$,

- if $\mathrm{k}=3.954 / 1.638$ and $S \leq 1.3937$ then $t=0.132 \sigma_{f}^{2}$.

To assess the influence of the coefficient of thermal expansion of outer side, the numerical results for two-layered model, are shown in Fig. 5. It is important to note, that the maximum stresses for homogeneity cylinder with $k=1$ don't exceed 1/3.

\section{Conclusion}

During the study of the thermal elasticity for a cylinder, with thin outer layer having a different coefficient of thermal expansion, the following results were obtained.

When a certain time value is reached, the nonlinear terms in the equations determining temperature and stress become negligibly small.

The non-stationarity of the problem of determining the temperature, and as a result of the stresses, is described by

\section{References}

[1] Yin, F., Rakita, M., Hu, S., Han, Q. "Overview of ultrasonic shot peening", Surface Engineering, 33(9), pp. 651-666, 2017. https://doi.org/10.1080/02670844.2017.1278838

[2] Benedict, G. F. "Thermal Energy Method: Deburring (TEM)", In: Nontraditional Manufacturing Processes, Routledge, Boca Raton, FL, USA, 2017, pp. 349-361. https://doi.org/10.1201/9780203745410-22

[3] Plankovskyy, S., Teodorczyk, A., Shypul, O., Tryfonov, O., Brega, D. "Determination of Detonable Gas Mixture Heat Fluxes at Thermal Deburring", Acta Polytechnica: Journal of Advanced Engineering, 59(2), pp. 162-169, 2019. https://doi.org/10.14311/AP.2019.59.0162

[4] Yi, J., Deng, Z., Zhou, W., Li, S. "Numerical Modeling of Transient Temperature and Stress in WC-10Co4Cr Coating During HighSpeed Grinding", International Journal of Precision Engineering and Manufacturing, 21(4), pp. 585-598, 2020.

https://doi.org/10.1007/s12541-019-00285-9

[5] Tschiptschin, A. P., Nishikawa, A. S., Varela, L. B., Pinedo, C. E. "Thermal stability of expanded austenite formed on a DC plasma nitrided 316L austenitic stainless steel", Thin Solid Films, 644, pp. 156-165, 2017. https://doi.org/10.1016/j.tsf.2017.06.065 a double piecewise function - the nonlinear when $t<0.25$ and the linear dependence otherwise.

The cumbersome analytical equations of the thermal elasticity problem is presented in the form of simple approximations.

Using obtained approximations, it is possible to calculate the application time of the required heat flux up to achievement the strength limitations of the treatment sets for the studied materials.

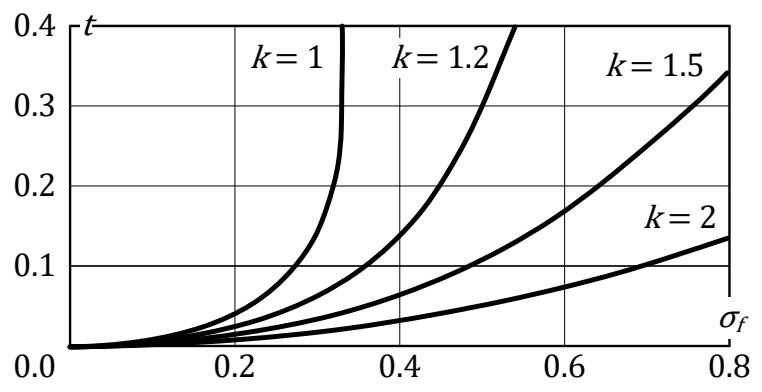

Fig. 5 Variation of the maximum stresses on the surface of the cylinder over the time for varied coefficients of thermal expansion in the outer layer

[6] Reddy, J. N., Chin, C. D. "Thermomechanical Analysis of Functionally Graded Cylinders and Plates", Journal of Thermal Stresses, 21(6), pp. 593-626, 1998. https://doi.org/10.1080/01495739808956165

[7] Grigorenko, A. Ya., Müller, W.H., Grigorenko, Y. M., Vlaikov, G. G. "Recent Developments in Anisotropic Heterogeneous Shell Theory: Applications of Refined and Three-dimensional Theory Volume IIB", Springer Nature, Singapore, Singapore, 2016. https://doi.org/10.1007/978-981-10-1596-0

[8] Li, W., Han, B. "Research and Application of Functionally Gradient Materials", IOP Conference Series: Materials Science and Engineering, 394(2), Article Number: 022065, 2018. https://doi.org/10.1088/1757-899X/394/2/022065

[9] Smahat, A., Megueni, A. "Numerical Modeling of an FG Plate Behavior Impacted at Low Velocity in a Temperature Field", Periodica Polytechnica Mechanical Engineering, 60(2), pp. 113-120, 2016. https://doi.org/10.3311/PPme.8734

[10] Biot, M. A. "Thermoelasticity and Irreversible Thermodynamics", Journal of Applied Physics, 27(3), pp. 240-253, 1956. https://doi.org/10.1063/1.1722351 
[11] Eraslan, A. N., Apatay, T. "Analytical solution to thermal loading and unloading of a cylinder subjected to periodic surface heating", Journal of Thermal Stresses, 39(8), pp. 928-941, 2016. https://doi.org/10.1080/01495739.2016.1189791

[12] Rakocha, I., Popovych, V. "The Mathematical Modeling and Investigation of the Stress-Strain State of the ThreeLayer Thermosensitive Hollow Cylinder", Acta Mechanica et Automatica, 10(3), pp. 181-188, 2016. https://doi.org/10.1515/ama-2016-0027

[13] Manthena, V. R., Srinivas, V. B., Kedar, G. D. "Analytical solution of heat conduction of a multilayered annular disk and associated thermal deflection and thermal stresses", Journal of Thermal Stresses, 43(5), pp. 563-578, 2020.

https://doi.org/10.1080/01495739.2020.1735975
[14] Evci, C., Gülgeç, M. "Functionally graded hollow cylinder under pressure and thermal loading: effect of material parameters on stress and temperature distributions", International Journal of Engineering Science, 123, pp. 92-108, 2018. https://doi.org/10.1016/j.ijengsci.2017.11.019

[15] Carslaw, H. S., Jaeger, J. C. "Conduction of heat in solids", Clarondon Press, Oxford, UK, 1959. 